\title{
The relationship between mobile phone location sensor data and depressive symptom severity
}

\author{
Sohrab Saeb ${ }^{1,2}$, Emily G Lattie ${ }^{1}$ ， Stephen M Schueller ${ }^{1}$ ， Konrad P Kording ${ }^{2}$ ， David C Mohr ${ }^{\text {Corresp. } 1}$ \\ 1 Department of Preventive Medicine, Northwestern University, Chicago, IL, United States \\ 2 Rehabilitation Institute of Chicago, Department of Physical Medicine and Rehabilitation, Northwestern University, Chicago, IL, United States \\ Corresponding Author: David C Mohr \\ Email address: d-mohr@northwestern.edu
}

Background. Smartphones offer the hope that depression can be detected using passively collected data from the phone sensors. The aim of this study was to replicate and extend previous work using geographic location (GPS) sensors to identify depressive symptom severity.

Methods. We used a dataset collected from 48 college students over a 10-week period, which included GPS phone sensor data and the Patient Health Questionnaire 9-item (PHQ-9) to evaluate depressive symptom severity at baseline and end-of-study. GPS features were calculated over the entire study, for weekdays and weekends, and in 2-week blocks.

Results. The results of this study replicated our previous findings that a number of GPS features, including location variance, entropy, and circadian movement, were significantly correlated with PHQ-9 scores ( $r$ 's ranging from -0.43 to $-0.46, p$-values $<.05$ ). We also found that these relationships were stronger when GPS features were calculated from weekend, compared to weekday, data. Although the correlation between baseline PHQ-9 scores with 2-week GPS features diminished as we moved further from baseline, correlations with the end-of-study scores remained significant regardless of the time point used to calculate the features.

Discussion. Our findings were consistent with past research demonstrating that GPS features may be an important and reliable predictor of depressive symptom severity. The varying strength of these relationships on weekends and weekdays suggests the role of weekend/weekday as a moderating variable. The finding that GPS features predict depressive symptom severity up to 10 weeks prior to assessment suggests that GPS features may have the potential as early warning signals of depression. 


\title{
The Relationship between Mobile Phone Location Sensor Data and Depressive Symptom Severity
}

\author{
Sohrab Saeb ${ }^{1,2}$, Emily Lattie ${ }^{1}$, Stephen M Schueller ${ }^{1}$, Konrad P Kording², David C Mohr ${ }^{1, *}$ \\ ${ }^{1}$ Center for Behavioral Intervention Technologies, Department of Preventive Medicine, Northwestern \\ University, Chicago, USA \\ ${ }^{2}$ Rehabilitation Institute of Chicago, Department of Physical Medicine and Rehabilitation, Northwestern \\ University, Chicago, USA \\ * Corresponding author: David C. Mohr, Professor at the Department of Preventive Medicine, \\ Northwestern University. Address: $10^{\text {th }}$ Floor, 750 N. Lake Shore Dr., Chicago, IL 60611, USA. Tel: 1- \\ 312-503-1403. Email: d-mohr@northwestern.edu
}


1

2

3

4

5

6

7

8

9

10

11

12

13

14

15

16

17

18

19

20

21

22

23

24

25

26

27

28

29

30

31

32

33

34

35

36

37

38

39

\section{ABSTRACT}

Background. Smartphones offer the hope that depression can be detected using passively collected data from the phone sensors. The aim of this study was to replicate and extend previous work using geographic location (GPS) sensors to identify depressive symptom severity. Methods. We used a dataset collected from 48 college students over a 10-week period, which included GPS phone sensor data and the Patient Health Questionnaire 9-item (PHQ-9) to evaluate depressive symptom severity at baseline and end-of-study. GPS features were calculated over the entire study, for weekdays and weekends, and in 2-week blocks.

Results. The results of this study replicated our previous findings that a number of GPS features, including location variance, entropy, and circadian movement, were significantly correlated with PHQ-9 scores ( $r$ 's ranging from -0.43 to -0.46 , $p$-values $<.05$ ). We also found that these relationships were stronger when GPS features were calculated from weekend, compared to weekday, data. Although the correlation between baseline PHQ-9 scores with 2-week GPS features diminished as we moved further from baseline, correlations with the end-of-study scores remained significant regardless of the time point used to calculate the features.

Discussion. Our findings were consistent with past research demonstrating that GPS features may be an important and reliable predictor of depressive symptom severity. The varying strength of these relationships on weekends and weekdays suggests the role of weekend/weekday as a moderating variable. The finding that GPS features predict depressive symptom severity up to 10 weeks prior to assessment suggests that GPS features may have the potential to serve as early warning signals of depression.

\section{INTRODUCTION}

Depression is common and debilitating, taking an enormous toll in terms of cost, morbidity, and mortality (Ferrari et al. 2013; Greenberg et al. 2015). The 12-month prevalence of major depressive disorder among adults in the US is 6.9\% (Kessler et al. 2005), and an additional 2-5\% have subsyndromal symptoms that warrant treatment (Ayuso-Mateos et al. 2010; Kessler et al. 1997). While treatments can be effective, failure to identify depression is a major factor in population-level disability. The US Preventive Services Task Force recommends annual screening for depression (Siu et al. 2016), and many argue that it should be far more frequent for at risk patients (Reynolds \& Frank 2016). Although screening alone has little effect on the management of depression by clinicians (Gilbody et al. 2008), even annual screening does not occur regularly, and only $37 \%$ of individuals with depression receive treatment in the first year of an episode. In fact, the median time to treatment in the US is 8 years (Wang et al. 2005). Furthermore, the Affordable Care Act and the Mental Health Parity Act not only require access to mental health services, but also the measurement of the quality of those services with symptom and functional outcomes (Basch et al. 2013). Thus, the healthcare system relies almost entirely on people with depression to present themselves and accurately report their symptoms, both to initiate treatment and for follow up. This is despite the fact that depressed individuals commonly experience loss of motivation, stigmatization, and a sense of hopelessness and 
helplessness (Mohr et al. 2010). Therefore, identification of patients experiencing treatable levels of depression in a timely manner is a substantial failure point in the healthcare system.

The mobile phone is arguably the most ubiquitous personal sensing device, with nearly twothirds $(64 \%)$ of adults in the United States owning smartphones (2015). These devices contain a growing complement of sensors which can provide data directly from the context of people's lives, and algorithms can translate phone sensor data into indicators of behavioral, social, and psychological targets. For example, Android provides activity status (walking, running, cycling, in vehicle, etc.) using the phone sensors. A growing body of research has demonstrated the potential of mobile phone sensors to detect a variety of behaviors related to depression, such as activity, sleep, and social interactions (Abdullah et al. 2014; Grunerbl et al. 2015; Kwapisz et al. 2011; Min et al. 2013; Shoaib et al. 2014; Thomée et al. 2011; Wiese et al. 2015). Thus, a mobile phone sensing platform that detects depression could transform the management of depression, allowing for continuous and ubiquitous diagnostics for at risk populations.

Detection of depression using phone sensors, however, is a much more difficult task than detecting behaviors that are observable and more proximal to the sensor data such as physical activity, sleep/wake patterns, and social interaction. Depression has a variety of symptoms and experiences that can include depressed mood, loss of interest, sleep disturbances, lack of energy, appetite disruption, trouble concentrating, psychomotor disturbances, feelings of hopelessness, guilt and worthlessness, social withdrawal, irritability, and suicidal thoughts (American Psychiatric Association 2013). While some of these symptoms might be captured through smartphone sensors, many cannot. Thus, there is no single set of sensors that is reliably and consistently sensitive to depression.

Detection of depression will likely require the development of features that translate mobile phone sensor data into behavioral targets that may be relevant to depression. We have recently done this with GPS data (Saeb et al. 2015a). Using GPS data collected from 28 participants over 2 weeks, we used a clustering algorithm to find each participant's favorite location. We then used these locations to calculate a number of features, including entropy, or the variability in the time spent across favorite locations, and circadian movement, or the periodicity of movement between those locations. We found a number of features to be significantly correlated with depressive symptoms severity. Therefore, while it is difficult to relate raw sensor data or their basic statistics directly to depression, their features are more likely to indicate the presence of depressive symptoms.

The purpose of this study is to replicate and extend our previous findings using a separate dataset. Replication is a critical step in the area of behavioral sensing. While exploration is essential to discovery, it is frequently done in small datasets. This can lead to spurious findings, as has recently been seen in the failure to replicate a widely cited paper (Likamwa et al. 2013) on the use of phone sensor data to detect mood (Ruwaard et al. 2016). Thus, the first aim was to replicate our previous findings on the relationship between GPS features and depression. Our second aim was to extend these findings by exploring those relationships in workdays and nonworkdays. Movement on workdays is likely determined to some degree by social roles and expectations, while movement on non-workdays is likely less determined by external demands 
and more by the individual's motivational state. We therefore hypothesized that GPS features would be more consistently related to depression on non-workdays, operationalized as weekends, than on workdays, operationalized as weekdays. The third aim was to explore the temporal directionality in the relationship between depression and GPS features.

\section{METHODS}

\section{Data}

We used a dataset from a study that was designed and conducted by researchers at Dartmouth College (Wang 2014). This dataset, known as StudentLife, was collected from 48 students of a computer science class for a duration of 10 weeks. The students consisted of 38 males and 10 females. Two of them were first-year, 14 second-year, 6 third-year, and 8 fourth-year Bachelor's students. There were also13 first-year and 1 second-year Master's student, and $3 \mathrm{PhD}$ students. Participants were racially diverse, with 23 Caucasians, 23 Asians, and 2 African-Americans. There was no inclusion or exclusion criteria based on the volunteers' mental health states. The authors of this paper had no involvement in the design of this study or the collection of these data. The study was approved by the Dartmouth College Institutional Review Board, and the study participants signed a consent form after being detailed about the type of data collected from their phones.

Data was collected using the StudentLife app, installed on Android devices. The students who did not own Android devices were provided with Google Nexus 4s phones for the period of the study. The StudentLife dataset consisted of a number of variables that were consistent with our previous study (Saeb et al. 2015a), including continuous phone GPS data and the Patient Health Questionnaire 9-item (PHQ-9) (Kroenke et al. 2001), a self-report measure of depression symptom severity. GPS data was collected continuously over 10 weeks, with a frequency of once in every 5 minutes. The PHQ-9 was administered at baseline and at the end of the study (followup).

Phone usage data in StudentLife was collected in a manner that was very different and not comparable to the data in our previous study (Saeb et al. 2015a): while we had logged every screen on/off event, they only logged these events when the phone lock duration was longer than 1 hour. We therefore focused our replication only on GPS.

The PHQ-9 scores were diverse across the participants and changed noticeably from baseline to follow-up, as shown in Figure 1. For 20 participants, the PHQ-9 scores decreased from baseline to follow-up, while for 16 they increased. However, only 7 participants had a change which was clinically meaningful, meaning that the absolute value of the change was greater than or equal to 5. 

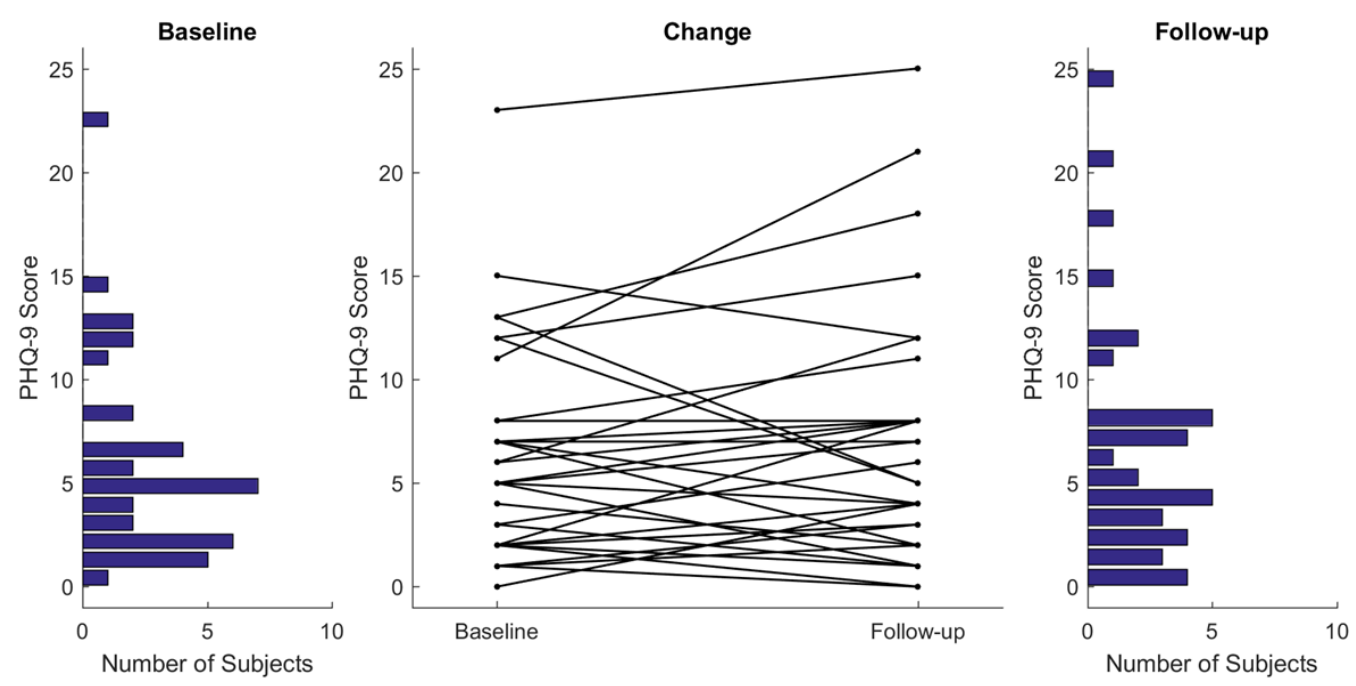

117 Figure 1. PHQ-9 score distribution at baseline (left) and the end of study follow-up (right). participant.

\section{0}

121

122

123

124

125

126

127

128

129

130

131

132

133

134

135

136

137

138

139

140

\section{Location Features}

We first extracted the spatial and temporal properties, or features, of the GPS sensor values. We used the same 8 features that we had used in our previous study (Saeb et al. 2015a), plus 3 new exploratory features: speed mean, speed variance, and raw entropy. Table 1 gives a brief description of all 11 features.

Each location feature was calculated in 3 different ways:

10-Week Features: We extracted these features from all GPS data collected during the entire 10 weeks of the study.

Weekday/Weekend Features: We extracted these features separately for workdays and nonworkdays. Because we did not have the participants' school or work schedules, we operationalized these as weekdays (Monday through Friday) and weekends (Saturday and Sunday). Then, we calculated weekday and weekend features separately from each of these two sets.

2-Week Features: To support analyses examining directionality of the correlations, we calculated these features from 2-week-long blocks of GPS data with an overlap of 1 week (Figure 2A). The 2-week period was selected as a block of time because a diagnosis of depression requires the presence of symptoms more days than not for 2 weeks. Therefore, we obtained 9 sets of features corresponding to 10 weeks. Similar to 10 -week features, we split these features into weekday and weekend features (Figure 2B).

Table 1. Features used in this study and their definitions. Features indicated with stars (*) are replicated from our previous study (Saeb et al. 2015a). 


\begin{tabular}{|c|c|}
\hline Feature & Definition \\
\hline Location Variance* & $\begin{array}{l}\text { Combined variance of latitude and longitude values: } \\
\text { Location Variance }=\log \left(\sigma_{\text {lat }}^{2}+\sigma_{\text {long }}^{2}\right) \\
\text { where } \sigma_{\text {lat }}^{2} \text { and } \sigma_{\text {long }}^{2} \text { are the variance of latitude and longitude, respectively. }\end{array}$ \\
\hline Circadian Movement* & $\begin{array}{l}\text { First, we used the least-squares spectral analysis (Press 2007) to obtain the } \\
\text { spectrum of the GPS signals. Then, we calculated the amount of energy that fell } \\
\text { into the frequency bins within a } 24 \pm 0.5 \text { hour period, in the following way: } \\
\qquad E=\frac{1}{i_{U}-i_{L_{i}}} \sum_{L}^{i_{L}} \operatorname{psd}\left(f_{i}\right) \\
\text { where psd }\left(f_{i}\right) \text { denotes the power spectral density at frequency bin } f_{i} \text {, and }{ }_{L} \text { and }{ }_{U} \\
\text { represent the lower and the upper bounds of the frequency range of interest, } \\
\text { corresponding to } 24.5 \text { and } 23.5 \text { hour periods respectively. We calculated } E \\
\text { separately for longitude and latitude, and obtained the total circadian movement as: } \\
\qquad C M=\log \left(E_{\text {lat }}+E_{\text {long }}\right)\end{array}$ \\
\hline Speed Mean & 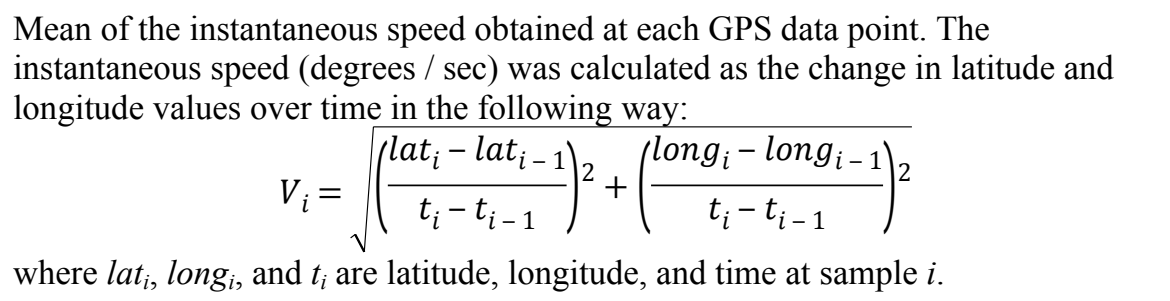 \\
\hline Speed Variance & Variance of the instantaneous speed. \\
\hline Total Distance* & $\begin{array}{l}\text { Total geographic displacement, as: } \\
\qquad \text { Total Distance }=\sum_{i} \sqrt{\left(\text { lat }_{i}-\text { lat }_{i-1}\right)^{2}+\left(\operatorname{long}_{i}-\operatorname{long}_{i-1}\right)^{2}} \\
\text { where } \text { lat }_{i} \text { and } \text { long }_{i} \text { show latitude and longitude values at sample } i \text {. }\end{array}$ \\
\hline Number of Clusters* & $\begin{array}{l}\text { Number of location clusters found by the adaptive } k \text {-means algorithm (Saeb et al. } \\
2015 \mathrm{a} \text { ). }\end{array}$ \\
\hline Entropy* & $\begin{array}{l}\text { Information theoretical entropy (Shannon 1997), which measured how each } \\
\text { participant's time was distributed over different location clusters: } \\
\qquad E n t r o p y=-\sum_{i=1}^{N} p_{i} \log \left(p_{i}\right) \\
\text { where } p_{i} \text { is the percentage of time spent at location } i \text {, and } N \text { is the total number of } \\
\text { location clusters. }\end{array}$ \\
\hline Normalized Entropy* & $\begin{array}{l}\text { Entropy normalized by the number of location clusters }(N) \text { : } \\
\qquad \text { Normalized Entropy }=\frac{\text { Entropy }}{\log (N)}\end{array}$ \\
\hline Raw Entropy & Same as entropy, with $p_{i}$ representing the number of data points in each latitude or \\
\hline
\end{tabular}




\begin{tabular}{|l|l|}
\hline & $\begin{array}{l}\text { longitude bin before clustering. A total number of } N=10 \text { bins was used. The total } \\
\text { raw entropy was defined as the sum of latitude and longitude raw entropies. }\end{array}$ \\
\hline Home Stay* & Percentage of time spent at home. \\
\hline Transition Time* & Percentage of time spent in transit, such as in a car or on bike. \\
\hline
\end{tabular}

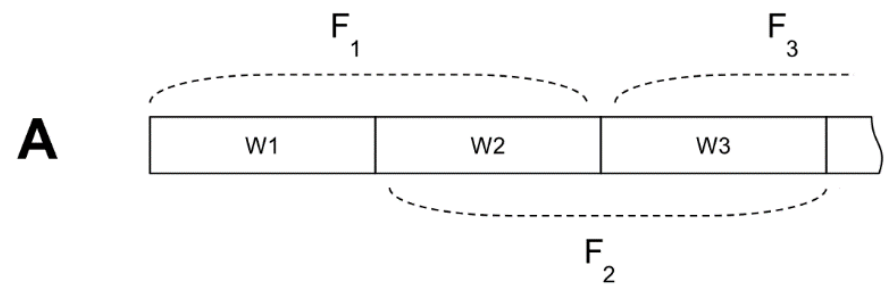

$\mathrm{F}_{\mathrm{WE}, 1}$

B

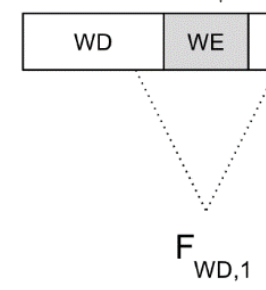

$\mathrm{F}_{\text {WE,2 }}$

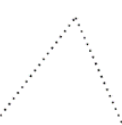

\begin{tabular}{l|l} 
WD & \\
& $\ddots$ \\
& $\ddots$ \\
& $\ddots$ \\
&
\end{tabular}

WE

\begin{tabular}{|l|l|l|} 
WD & WE & \\
\hline
\end{tabular}

Figure 2. Feature extraction procedure for 2 -week features. (A) The first set of features $\left(F_{1}\right.$ to $F_{9}$ ) were extracted from 2 -week blocks of sensor data that had an overlap of 1 week. (B) The second set of features were extracted after each week of data was split into weekday (Monday to Friday) and weekend (Saturday and Sunday). Weekday features $\left(F_{W D, 1}\right.$ to $\left.F_{W D, 9}\right)$ were extracted from the weekday part and weekend features $\left(F_{W E, I}\right.$ to $\left.F_{W E, 9}\right)$ from the weekend part of each 2 -week block.

\section{Data Analysis}

We evaluated the relationship between each set of features (10-week and 2-week, each for all days, weekends, or weekdays) and depressive symptoms severity as measured by the PHQ-9. We used linear correlation coefficient $(r)$ and considered $p<0.05$ as the significance level. In order to reduce the possibility that results were generated by chance, we created 1000 bootstrap subsamples (Efron \& Tibshirani 1993) to estimate these correlation coefficients and their 95\% confidence intervals (CIs). We only considered those coefficients significant for which the $95 \%$ CI of their associated $p$-values fell below 0.05 . Correlation analysis was conducted for baseline and follow-up PHQ-9 scores separately. In addition, although there were only 7 participants who had clinically meaningful change $(\geq 5)$ in their PHQ-9 scores, we also performed a correlation 
159 analysis with the change in PHQ-9 in order to see if any of the score changes, clinically

160 meaningful or not, were related to GPS features.

\section{RESULTS}

\section{2}

163

164

165

166

167

168

169

170

\section{0-Week Features}

The results of the correlation analysis between 10-week location features and depression scores are shown in Table 2. Results reaching the significance level (see Data Analysis) are shown in bold. Particularly, location variance, circadian movement, entropy, home stay, and number of clusters all had absolute correlation coefficients $|r| \geq 0.4$ with the follow-up PHQ-9 scores. Correlations between these features and baseline scores were weaker, and none of them were statistically significant. Overall, these results were consistent with our previous findings (Saeb et al. 2015a).

Table 2. Linear correlation coefficients (r) between individual 10-week features and PHQ-9 scores, and their $95 \%$ confidence intervals. Features indicated with stars $(*)$ are replicated from our previous study (Saeb et al. 2015a). Bold values indicate significant correlations.

\begin{tabular}{|l|l|l|l|}
\hline Feature & Baseline $(\boldsymbol{n}=\mathbf{4 6})$ & Follow-up $(\mathbf{n}=\mathbf{3 8})$ & Change $(\mathbf{n}=\mathbf{3 8})$ \\
\hline Location Variance* & $-0.29 \pm 0.008$ & $\mathbf{- 0 . 4 3} \pm \mathbf{0 . 0 0 7}$ & $-0.34 \pm 0.008$ \\
\hline Circadian Movement* & $-0.34 \pm 0.006$ & $\mathbf{- 0 . 4 8} \pm \mathbf{0 . 0 0 6}$ & $-0.33 \pm 0.009$ \\
\hline Speed Mean & $-0.03 \pm 0.007$ & $-0.06 \pm 0.005$ & $0.04 \pm 0.008$ \\
\hline Speed Variance & $-0.07 \pm 0.007$ & $-0.06 \pm 0.005$ & $0.06 \pm 0.007$ \\
\hline Total Distance* & $-0.23 \pm 0.004$ & $-0.18 \pm 0.006$ & $-0.03 \pm 0.006$ \\
\hline Number of Clusters* & $-\mathbf{0 . 3 8} \pm \mathbf{0 . 0 0 5}$ & $\mathbf{- 0 . 4 4} \pm \mathbf{0 . 0 0 4}$ & $-0.24 \pm 0.007$ \\
\hline Entropy* & $-0.31 \pm 0.007$ & $\mathbf{- 0 . 4 6} \pm \mathbf{0 . 0 0 5}$ & $-0.28 \pm 0.008$ \\
\hline Normalized Entropy* & $-0.26 \pm 0.007$ & $\mathbf{- 0 . 4 4} \pm \mathbf{0 . 0 0 5}$ & $-0.30 \pm 0.009$ \\
\hline Raw Entropy & $0.17 \pm 0.009$ & $0.22 \pm 0.008$ & $0.15 \pm 0.010$ \\
\hline Home Stay* & $0.22 \pm 0.008$ & $\mathbf{0 . 4 3} \pm \mathbf{0 . 0 0 5}$ & $0.30 \pm 0.009$ \\
\hline Transition Time* & $-0.30 \pm 0.006$ & $-0.32 \pm 0.005$ & $-0.12 \pm 0.009$ \\
\hline
\end{tabular}

\section{Weekday/Weekend Features}

174 The results of the correlations between GPS features and depression by weekday and weekend 175 are shown in Table 3. All of those 10-week features that were significantly related to PHQ-9 
176 scores (see Table 2) were also significant when calculated from weekends, whereas only

177 normalized entropy was significantly related to the scores as a weekday feature. The magnitude

178 of the relationship between weekend features and PHQ-9 scores was larger than the magnitude of

179 the relationship between 10-week features and PHQ-9 scores. However, given the small sample

180 size, we were not adequately powered to test if these differences were significant.

181 Table 3. Linear correlation coefficients $(r)$ between individual weekend and weekday 182 features and PHQ-9 scores, and their 95\% confidence intervals. Bold values indicate 183 significant correlations (see Data Analysis).

\begin{tabular}{|c|c|c|c|c|c|c|}
\hline \multirow[t]{2}{*}{ Feature } & \multicolumn{3}{|l|}{ Weekday } & \multicolumn{3}{|l|}{ Weekend } \\
\hline & $\begin{array}{l}\text { Baseline } \\
(n=46)\end{array}$ & $\begin{array}{l}\text { Follow-up } \\
(n=38)\end{array}$ & $\begin{array}{l}\text { Change } \\
(n=38)\end{array}$ & $\begin{array}{l}\text { Baseline } \\
(n=46)\end{array}$ & $\begin{array}{l}\text { Follow-up } \\
(n=38)\end{array}$ & $\begin{array}{l}\text { Change } \\
(n=38)\end{array}$ \\
\hline Location Variance & $\begin{array}{l}-0.15 \\
\pm 0.008\end{array}$ & $\begin{array}{l}-0.20 \\
\pm 0.008\end{array}$ & $\begin{array}{l}-0.22 \\
\pm 0.009\end{array}$ & $\begin{array}{l}-0.31 \\
\pm 0.008\end{array}$ & $\begin{array}{l}-\mathbf{0 . 4 7} \\
\pm \mathbf{0 . 0 0 7}\end{array}$ & $\begin{array}{l}-0.39 \\
\pm 0.008\end{array}$ \\
\hline Circadian Movement & $\begin{array}{l}-0.22 \\
\pm 0.007\end{array}$ & $\begin{array}{l}-0.28 \\
\pm 0.008\end{array}$ & $\begin{array}{l}-0.25 \\
\pm 0.009\end{array}$ & $\begin{array}{l}-0.35 \\
\pm 0.007\end{array}$ & $\begin{array}{l}-\mathbf{- 0 . 5 1} \\
\pm 0.006\end{array}$ & $\begin{array}{l}-0.36 \\
\pm 0.008\end{array}$ \\
\hline Speed Mean & $\begin{array}{l}-0.00 \\
\pm 0.008\end{array}$ & $\begin{array}{l}-0.06 \\
\pm 0.005\end{array}$ & $\begin{array}{l}0.03 \\
\pm 0.008\end{array}$ & $\begin{array}{l}-0.13 \\
\pm 0.005\end{array}$ & $\begin{array}{l}-0.06 \\
\pm 0.006\end{array}$ & $\begin{array}{l}0.05 \\
\pm 0.009\end{array}$ \\
\hline Speed Variance & $\begin{array}{l}-0.05 \\
\pm 0.008\end{array}$ & $\begin{array}{l}-0.07 \\
\pm 0.005\end{array}$ & $\begin{array}{l}0.02 \\
\pm 0.007\end{array}$ & $\begin{array}{l}-0.13 \\
\pm 0.004\end{array}$ & $\begin{array}{l}-0.05 \\
\pm 0.006\end{array}$ & $\begin{array}{l}0.10 \\
\pm 0.008\end{array}$ \\
\hline Total Distance & $\begin{array}{l}-0.20 \\
\pm 0.004\end{array}$ & $\begin{array}{l}-0.15 \\
\pm 0.005\end{array}$ & $\begin{array}{l}-0.01 \\
\pm 0.006\end{array}$ & $\begin{array}{l}-0.25 \\
\pm 0.004\end{array}$ & $\begin{array}{l}-0.20 \\
\pm 0.005\end{array}$ & $\begin{array}{l}-0.03 \\
\pm 0.006\end{array}$ \\
\hline Number of Clusters & $\begin{array}{l}-0.19 \\
\pm 0.006\end{array}$ & $\begin{array}{l}-0.25 \\
\pm 0.005\end{array}$ & $\begin{array}{l}-0.14 \\
\pm 0.008\end{array}$ & $\begin{array}{l}-0.34 \\
\pm 0.006\end{array}$ & $\begin{array}{l}-0.46 \\
\pm 0.004\end{array}$ & $\begin{array}{l}-0.32 \\
\pm 0.007\end{array}$ \\
\hline Entropy & $\begin{array}{l}-0.21 \\
\pm 0.007\end{array}$ & $\begin{array}{l}-0.34 \\
\pm 0.006\end{array}$ & $\begin{array}{l}-0.20 \\
\pm 0.009\end{array}$ & $\begin{array}{l}-0.30 \\
\pm 0.008\end{array}$ & $\begin{array}{l}-\mathbf{0 . 5 5} \\
\pm 0.004\end{array}$ & $\begin{array}{l}-0.38 \\
\pm 0.008\end{array}$ \\
\hline Normalized Entropy & $\begin{array}{l}-0.21 \\
\pm 0.008\end{array}$ & $\begin{array}{l}-\mathbf{0 . 3 9} \\
\pm 0.006\end{array}$ & $\begin{array}{l}-0.24 \\
\pm 0.009\end{array}$ & $\begin{array}{l}-0.28 \\
\pm 0.008\end{array}$ & $\begin{array}{l}-\mathbf{0 . 5 4} \\
\pm 0.004\end{array}$ & $\begin{array}{l}-0.41 \\
\pm 0.009\end{array}$ \\
\hline Raw Entropy & $\begin{array}{l}0.05 \\
\pm 0.008\end{array}$ & $\begin{array}{l}-0.04 \\
\pm 0.008\end{array}$ & $\begin{array}{l}0.01 \\
\pm 0.010\end{array}$ & $\begin{array}{l}0.04 \\
\pm 0.008\end{array}$ & $\begin{array}{l}-0.01 \\
\pm 0.008\end{array}$ & $\begin{array}{l}0.03 \\
\pm 0.009\end{array}$ \\
\hline Home Stay & $\begin{array}{l}0.19 \\
\pm 0.008\end{array}$ & $\begin{array}{l}0.37 \\
\pm 0.006\end{array}$ & $\begin{array}{l}0.23 \\
\pm 0.009\end{array}$ & $\begin{array}{l}0.23 \\
\pm 0.007\end{array}$ & $\begin{array}{l}0.50 \\
\pm 0.004\end{array}$ & $\begin{array}{l}0.35 \\
\pm 0.008\end{array}$ \\
\hline Transition Time & $\begin{array}{l}-0.27 \\
\pm 0.006\end{array}$ & $\begin{array}{l}-0.29 \\
\pm 0.006\end{array}$ & $\begin{array}{l}-0.14 \\
\pm 0.010\end{array}$ & $\begin{array}{l}-0.36 \\
\pm 0.006\end{array}$ & $\begin{array}{l}-0.32 \\
\pm 0.008\end{array}$ & $\begin{array}{l}-0.06 \\
\pm 0.009\end{array}$ \\
\hline
\end{tabular}

\section{2-Week Features}

185 Finally, we examined how 2-week GPS features obtained at different times during the study correlated with baseline and follow-up depression scores. These analyses were performed only 
187

188

189

190

191

192

193

194

195

196

197

198

199

200

201

202

203

204

205

206

207

208

209

210

211

212

213

214

215

216

217

218

219

220

221

222

223

224

on those features that showed significant relationships with PHQ-9 scores in the previous section (see Tables 2 and 3). We ran these analyses separately for weekday and weekend features, calculated at each week.

Consistent with the results in Table 3, correlations of weekend features (Figure 3C-D) were generally stronger than weekday features (Figure 3A-B). Furthermore, correlations between baseline PHQ-9 scores were significantly associated with weekend location features in the first weeks immediately following depression assessment, but quickly became non-significant and approached zero at the end of the 10 weeks (Figure 3C). In contrast, correlations between weekend location features and the follow-up PHQ-9 scores generally remained significant regardless of the time point at which features were extracted (Figure 3D). This was also true for 3 out of the 6 features extracted on weekdays (Figure 3B), including homestay, entropy, and normalized entropy. Overall, while these results were consistent with correlation results in Table 3 , they indicated that these correlations changed over time.

\section{DISCUSSION}

In this study, we were able to replicate our previous findings of the relationship between severity of depressive symptoms and GPS location features, including location variance, entropy, and circadian movement (Saeb et al. 2015a; Saeb et al. 2015b), in the StudentLife dataset (Wang 2014). This is particularly notable as the StudentLife dataset was collected completely independently of this research group, using a very different sample that consisted of college students in a small town as opposed to the general population in a large city (Chicago) from our first study. Furthermore, subsequent to our initial publication, a third independent group, Canzian and Musolesi, developed very similar location features and had similar findings (Canzian \& Musolesi 2015). Together, these findings provide strong evidence that features of GPS location such as the number of places a person goes, how time is spent in these locations, and the circadian rhythm of movement through geographic space, are strongly related to the severity of depressive symptoms.

Replication in the field of psychological science is important given how frequently replication studies fail. In fact, a recent meta-study showed that only one-third to one-half of experimental results reported in psychological science were replicated in later studies (Open Science 2015). This is particularly crucial in the field of behavioral sensing using smartphones since studies in this field frequently use small samples, are usually not cross-validated, and failure to replicate has already been observed (Ruwaard et al. 2016). Thus, replication studies such as the present one can be seen as the studies that make findings real.

In addition to replication, we took this opportunity to extend previous findings in two important ways. First, we examined the relation of GPS features to depression on weekends and weekdays separately. We suspected that the relationship between GPS features and depression seen in our first study might reflect participants' motivational states. If true, we hypothesized that the signal would be stronger when the individual had more control over their own movement, such as on a 
non-workday, than when movement was in response to external demands, such as on workdays when a larger percentage of movement is typically determined by job school-related demands. Indeed, we did see larger correlations on weekends between many of the GPS features and depression, than on weekdays.
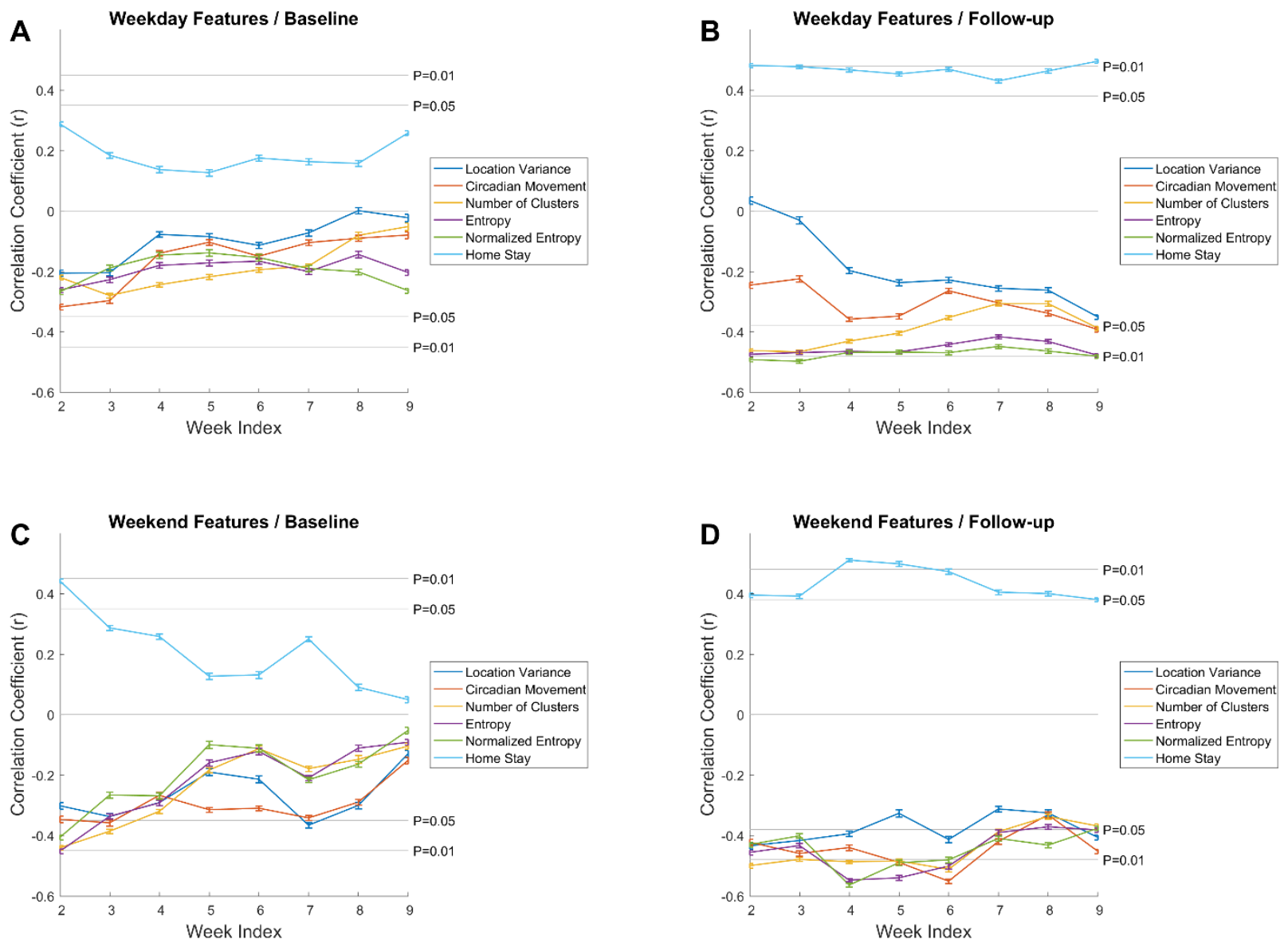

Figure 3. Mean temporal correlations between 2-week location features, calculated at different time points during the study, and baseline and follow-up PHQ-9 scores. Error bars show the $95 \%$ confidence intervals. In (A-B), features were obtained from weekday data only, and in (C-D), they were extracted from weekend sensor data. For each 2-week feature set, week indices indicate when the 2 -week period ended. Due to sparsity of data in week 10, we excluded it from this analysis.

The weekend vs. weekday finding illustrates the importance of considering a wide range of possible features or conditions that improve sensor-based detection of behaviors and mental health conditions. A large empirical and theoretical literature has focused on the complex environmental, situational, personality, and motivational factors that drive human behavior (Fleeson 2004; Mischel 1969; Rauthmann et al. 2014). Consideration of such complex interactions likely has considerable value in the development of sensor-based detection of 
242 behavior and mental health conditions. For example, simply looking at these GPS data, one

243

244

245

246

247

248

249

250

251

252

253

254

255

256

257

258

259

260

261

262

263

264

265

266

267

268

269

270

271

272

273

274

275

276

277

278

279

280

281

might imagine a wide variety of features to improve the detection, such as urban versus rural, northern versus southern, and economically deprived versus wealthy locations, as well as season, weather, and climate. Such features can also be detected passively, and in combination they may improve the detection accuracy.

The second way we extended our previous findings was to examine the temporal relationship between GPS features and depression. The StudentLife study extended over 10 weeks, as compared to 2 weeks for our first study, making it a better dataset to explore temporal relationships. The relationship between depressive symptom severity and subsequent location features was significant for the first weeks, but then rapidly declined over time. This was most notable when using the weekend location features, which were generally stronger predictors. In contrast, the correlations between weekend location features immediately prior to the assessment of depression symptom severity were very similar to the correlation 8-10 weeks prior. This suggests that location features may be early warning detectors of depression.

It is also interesting to note that some location features, such as location variance and circadian movement, which were strongly predictive of depression 8-10 weeks prior when measured on weekends, showed no significant prediction when measured on weekdays. Nevertheless, weekday measurement did become stronger the closer their extraction occurred to the assessment. This may suggest that depression-related disruption in movement first manifests itself only when there are no social constraints (e.g., on non-workdays), but as the disruption in GPS features shifts to manifest itself on workdays, risk of subsequent depression becomes more immediate. Thus, the relationship between features over time may improve the prediction. For example, the weekend features 10 weeks prior, combined with weekday features at a later date may represent the shift of risk factors occurring in the absence of social demands to occurrence in the presence of social demands, thereby improving the prediction of depression. Such hypotheses can be tested in larger datasets.

There are a number of limitations that should be noted as well. First, the findings on weekend and weekday features and temporal relationships contain a large number of correlation analyses which introduce the possibility of alpha slippage, particularly for the exploratory analyses. Furthermore, we were not sufficiently powered to detect workday/non-workday differences in the relationship between GPS and depression, nor the differences in these relationships over time. Thus, these findings should be considered preliminary until replicated. In contrast, given the consistency across studies and populations, the number of analyses for the replication analyses is not a significant limitation for this study.

Second, the distinction between weekdays and weekend is not necessarily a defining feature for every person. Some schools might have schedules that do not fall cleanly into a 5-day work week and many people clearly work on weekends. In a previous analysis of the StudentLife dataset, for example, specific aspects about Dartmouth social behavior was used to explore typical "party" days which did not line up exactly with weekend/weekdays (Wang et al. 2015).

Weekend/weekday was simply an operationalization for workday/non-workday. In a broader, 
282

283

284

285

286

287

288

289

290

291

292

293

294

295

296

297

298

299

300

301

302

303

304

305

306

307

308

309

310

311

312

313

314

315

316

317

318

non-student sample, this might be detected passively for most individuals by identifying their work location.

Finally, this study had a relatively small sample size. Our finding of the importance of workday/non-workday distinction highlights the likely importance of the variables that moderate the relationship between sensor data and mental health. There are likely many such variables, such as climate, age, and urban/rural locations, to name a few. Therefore, the development of effective behavioral sensing prediction models for mental health will likely require a much larger sample size. Furthermore, behavioral sensing studies - including ours - require longer periods of time to capture the slow changes in the disease state and in the behavioral features. Most studies of behavioral sensing in depression only have 2 or 3 assessment points for each individual, which makes the dataset underpowered for investigating the longitudinal relationship between sensor data - which has much higher frequency - and depression. Longer studies with more frequent assessments of depressive symptoms would allow researchers to more accurately model and predict these changes, both across the population and within each individuals.

In conclusion, our study supports the potential of smartphone sensor technology in providing biomarkers of depression in daily life. However, even with strong biomarkers, it remains a challenge to develop a system that can passively detect depression with low false positive and false negative rates. For this potential to be realized, well-designed studies with large numbers of participants over longer periods of time will be required. Such properly designed studies have the potential to transform mental health care, allowing for objective, ubiquitous, sensor-based evaluations that require little to no ongoing effort to estimate the risk of depression.

\section{ACKNOWLEDGEMENT}

The authors would like to thank Dr. Andrew T. Campbell for helpful discussions on the StudentLife dataset and for providing information on Dartmouth College Institutional Review Board approval.

\section{REFERENCES}

2015. The Smartphone Difference. http://www.pewinternet.org/2015/04/01/us-smartphone-use-in2015/: Pew Research Center.

Abdullah S, Matthews M, Murnane EL, Gay G, and Choudhury T. 2014. Towards circadian computing: "early to bed and early to rise" makes some of us unhealthy and sleep deprived. Proceedings of the 2014 ACM International Joint Conference on Pervasive and Ubiquitous Computing. Seattle, Washington: ACM. p 673-684.

American Psychiatric Association AP. 2013. Diagnostic and statistical manual of mental disorders (DSM$\left.5^{\circledR}\right)$ : American Psychiatric Pub.

Ayuso-Mateos JL, Nuevo R, Verdes E, Naidoo N, and Chatterji S. 2010. From depressive symptoms to depressive disorders: the relevance of thresholds. Br J Psychiatry 196:365-371. 10.1192/bjp.bp.109.071191 
319

Basch E, Torda P, and Adams K. 2013. Standards for patient-reported outcome-based performance measures. JAMA 310:139-140. 10.1001/jama.2013.6855

Canzian L, and Musolesi M. 2015. Trajectories of depression: unobtrusive monitoring of depressive states by means of smartphone mobility traces analysis. Proceedings of the 2015 ACM International Joint Conference on Pervasive and Ubiquitous Computing. Osaka, Japan: ACM. p 1293-1304.

Efron B, and Tibshirani R. 1993. An introduction to the bootstrap. New York: Chapman \& Hall.

Ferrari AJ, Charlson FJ, Norman RE, Patten SB, Freedman G, Murray CJ, Vos T, and Whiteford HA. 2013. Burden of depressive disorders by country, sex, age, and year: findings from the global burden of disease study 2010. PLoS Med 10:e1001547. 10.1371/journal.pmed.1001547

Fleeson W. 2004. Moving personality beyond the person-situation debate - The challenge and the opportunity of within-person variability. Current Directions in Psychological Science 13:83-87. DOI 10.1111/j.0963-7214.2004.00280.x

Gilbody S, Sheldon T, and House A. 2008. Screening and case-finding instruments for depression: a meta-analysis. CMAJ 178:997-1003. 10.1503/cmaj.070281

Greenberg PE, Fournier AA, Sisitsky T, Pike CT, and Kessler RC. 2015. The economic burden of adults with major depressive disorder in the United States (2005 and 2010). J Clin Psychiatry 76:155-162. 10.4088/JCP.14m09298

Grunerbl A, Muaremi A, Osmani V, Bahle G, Ohler S, Troster G, Mayora O, Haring C, and Lukowicz P. 2015. Smartphone-based recognition of states and state changes in bipolar disorder patients. IEEE J Biomed Health Inform 19:140-148. 10.1109/JBHI.2014.2343154

Kessler RC, Chiu WT, Demler O, Merikangas KR, and Walters EE. 2005. Prevalence, severity, and comorbidity of 12-month DSM-IV disorders in the National Comorbidity Survey Replication. Arch Gen Psychiatry 62:617-627. 10.1001/archpsyc.62.6.617

Kessler RC, Zhao S, Blazer DG, and Swartz M. 1997. Prevalence, correlates, and course of minor depression and major depression in the National Comorbidity Survey. J Affect Disord 45:19-30.

Kroenke K, Spitzer RL, and Williams JBW. 2001. The PHQ-9 - Validity of a brief depression severity measure. Journal of General Internal Medicine 16:606-613. DOI 10.1046/j.15251497.2001.016009606.x

Kwapisz JR, Weiss GM, and Moore SA. 2011. Activity recognition using cell phone accelerometers. SIGKDD Explor News/ 12:74-82. 10.1145/1964897.1964918

Likamwa R, Liu Y, Lane ND, Zhong L. 2013. MoodScope: Building a Mood Sensor from Smartphone Usage Patterns. MobiSys'13. Taipei, Taiwan: ACM.

Min J-K, Wiese J, Hong JI, and Zimmerman J. 2013. Mining smartphone data to classify life-facets of social relationships. Proceedings of the 2013 conference on Computer supported cooperative work. San Antonio, Texas, USA: ACM. p 285-294.

Mischel W. 1969. Continuity and change in personality. Am Psychol 24:1012-1018.

Mohr DC, Ho J, Duffecy J, Baron KG, Lehman KA, Jin L, and Reifler D. 2010. Perceived barriers to psychological treatments and their relationship to depression. J Clin Psychol 66:394-409. 10.1002/jclp.20659

Open Science C. 2015. PSYCHOLOGY. Estimating the reproducibility of psychological science. Science 349:aac4716. 10.1126/science.aac4716

Press WH. 2007. Numerical recipes : the art of scientific computing. Cambridge, UK ; New York: Cambridge University Press.

Rauthmann JF, Gallardo-Pujol D, Guillaume EM, Todd E, Nave CS, Sherman RA, Ziegler M, Jones AB, and Funder DC. 2014. The Situational Eight DIAMONDS: A Taxonomy of Major Dimensions of Situation Characteristics. Journal of Personality and Social Psychology 107:677-718. $10.1037 / \mathrm{a} 0037250$ 
367

Reynolds CF, 3rd, and Frank E. 2016. US Preventive Services Task Force Recommendation Statement on Screening for Depression in Adults: Not Good Enough. JAMA Psychiatry 73:189-190. 10.1001/jamapsychiatry.2015.3281

Ruwaard J, Ejdys M, Schrader N, Sijbrandij M, and Riper H. 2016. Mobile Phone-Based Unobtrusive Ecological Momentary Assessment of Day-to-Day Mood: An Explorative Study. Journal of Medical Internet Research 18:68-68.

Saeb S, Zhang M, Karr CJ, Schueller SM, Corden ME, Kording KP, and Mohr DC. 2015a. Mobile Phone Sensor Correlates of Depressive Symptom Severity in Daily-Life Behavior: An Exploratory Study. Journal of Medical Internet Research 17. ARTN e17510.2196/jmir.4273

Saeb S, Zhang M, Kwasny MM, Karr CJ, Konrad, and Mohr DC. 2015b. The Relationship between Clinical, Momentary, and Sensor-based Assessment of Depression. 9th International Conference on Pervasive Computing Technologies for Healthcare (Pervasive Health). Istanbul, Turkey.

Shannon CE. 1997. The mathematical theory of communication. 1963. MD Comput 14:306-317.

Shoaib M, Bosch S, Incel OD, Scholten H, and Havinga PJ. 2014. Fusion of smartphone motion sensors for physical activity recognition. Sensors (Basel) 14:10146-10176. 10.3390/s140610146

Siu AL, Force USPST, Bibbins-Domingo K, Grossman DC, Baumann LC, Davidson KW, Ebell M, Garcia FA, Gillman M, Herzstein J, Kemper AR, Krist AH, Kurth AE, Owens DK, Phillips WR, Phipps MG, and Pignone MP. 2016. Screening for Depression in Adults: US Preventive Services Task Force Recommendation Statement. JAMA 315:380-387. 10.1001/jama.2015.18392

Thomée S, Härenstam A, and Hagberg M. 2011. Mobile phone use and stress, sleep disturbances, and symptoms of depression among young adults-a prospective cohort study. BMC public health 11:1.

Wang PS, Berglund P, Olfson M, Pincus HA, Wells KB, and Kessler RC. 2005. Failure and delay in initial treatment contact after first onset of mental disorders in the National Comorbidity Survey Replication. Arch Gen Psychiatry 62:603-613. 10.1001/archpsyc.62.6.603

Wang R, Fanglin Chen, Zhenyu Chen, Tianxing Li, Gabriella Harari, Stefanie Tignor, Xia Zhou, Dror BenZeev, and Andrew T. Campbell. 2014. StudentLife: assessing mental health, academic performance and behavioral trends of college students using smartphones. 2014 ACM International Joint Conference on Pervasive and Ubiquitous Computing: ACM. p 3-14.

Wang R, Harari G, Hao P, Zhou X, and Campbell AT. 2015. SmartGPA: how smartphones can assess and predict academic performance of college students. Proceedings of the 2015 ACM International Joint Conference on Pervasive and Ubiquitous Computing. Osaka, Japan: ACM. p 295-306.

Wiese J, Min J-K, Hong JI, and Zimmerman J. 2015. "You Never Call, You Never Write": Call and SMS Logs Do Not Always Indicate Tie Strength. Proceedings of the 18th ACM Conference on Computer Supported Cooperative Work \&\#38; Social Computing. Vancouver, BC, Canada: ACM. p 765-774. 